\title{
Real time numerical reconstruction of digitally recorded holograms in digital in-line holographic microscopy by using a graphics processing unit
}

\author{
C. Trujillo, John F. Restrepo and J. Garcia-Sucerquia* \\ School of Physics, Universidad Nacional de Colombia Sede Medellin, A.A. 3840, Medellín - Colombia
}

Received November 30, 2010; accepted December 17, 2010; published December 31, 2010

\begin{abstract}
Digital in-line holographic microscopy (DIHM) is perhaps the simplest methodology to obtain three-dimensional information from the micrometre world. The recovery of complex amplitude scattered by the specimens, relies on quite robust algorithms that consume large amounts of time. In this letter is presented the numerical reconstruction in real-time of holograms acquired in DIHM. The use of a graphics processing unit combine with a reduced number of operations allows for reconstructing DIHM holograms of $1024 \times 1024$ pixels up to 32 frames per second.
\end{abstract}

In the field of numerical reconstruction of digitally recorded holograms, named digital holography $(\mathrm{DH})$, the simplest and most versatile development is digital in-line holographic microscopy (DHIM) [1]. Because of its simplicity and robustness, this microscopy methodology has been applied to biology, material sciences and different studies where the tracking of particles is required in three spatial dimensions and time [2]. Currently, this tracking process is done by means of post processing thousands of previously recorded holograms; this extremely time consuming process, which often does not lead to successful outcomes, constitutes an important bottle neck for a wider application of DIHM. To overcome this setback, many efforts have been made in order to reduce the reconstruction time of DIHM holograms; the most remarkable one has led to a clever solution of the diffraction integral that constitutes the corner stone of this microscopy method [3]. Currently, this outstanding numerical implementation can be powered with capabilities provided by the graphics processing units (GPU); this implementation could allow the reconstruction of DIHM holograms at almost video rate.

The accelerated development of the GPU, pushed forward by the huge market of video games, has provided incredible tools for video game lovers. Because the key of a realistic video game is the capability of doing thousands of floating point operations in the shortest possible time, the GPUs perfectly fit in many problems of physics [4]. The field of optics has not been excluded from these developments; in particular, the GPUs have been already used for accelerating the reconstruction of digitally

*E-mail:jigarcia@unal.edu.co recorded holograms [5]; the authors used a GPU GeForce $8800 \mathrm{GTS}$ operating at $1.2 \mathrm{GHz}$ with 96 stream processors and a memory of $640 \mathrm{MB}$. With this hardware they can reconstruct holograms of $512 \times 512$ pixels at a rate of 24 frames per second (fps); because of the off-axis configuration, the hologram reconstruction can be performed by the Fresnel transform or the convolution approach [6]. In DIHM, two particles that are $\Delta r$ apart can be resolved if $\Delta r \geq \lambda / 2 N A$ [1], with $\lambda$ - the illumination wavelength and $N A$ - the numerical aperture of the system. Because DIHM is a lensless holographic microscopy technique, the $N A$ is controlled by the width $W$ of the recording device and its distance $L$ to the illumination source, namely $N A=1 / \sqrt{1+4 W^{2} / L^{2}}$. This condition imposes that in order to achieve micrometer resolution in DIHM with visible light; the width of the recording device must be of the order of its distance to the point source. As the numerical reconstruction of holograms relies on the computation of the diffraction integral in discrete coordinates, the limits of application of these algorithms [7] dictate that in practical applications DIHM holograms have at least 1024x1024 pixels. Notwithstanding the DIHM oriented implementation of the diffraction integral [3], the reconstruction of holograms with $2048 \times 2048$ pixels takes several seconds, as performed in a modern personal computer; this deferred time discourages the application of DIHM in many fields.

In DIHM the hologram reconstruction can be described as the diffraction that a converging spherical wavefront undergoes as it illuminates the recorded hologram. In the Fraunhofer domain, the above described diffraction process is precisely described by the Kirchoff-Helmholtz transform $[1,3]$ :

$$
K(\xi)=\int_{\sigma} \tilde{I}(\mathbf{r}) \exp [i k(\xi \cdot \mathbf{r} /|\mathbf{r}|)] d^{2} r
$$

In Eq. (1) the integration extends over the surface of the screen $\sigma$, the CCD or CMOS camera, with coordinates $\mathbf{r}=(x, y, L), L$ the distance from the pinhole to the center 
of the screen; $k=2 \pi / \lambda$ is the wave number; $\tilde{I}(\mathbf{r})$ is the contrast in-line hologram obtained by subtracting the images with and without the sample present; and $\xi=\left(\xi_{x}, \xi_{y}, z_{r}\right)$ are the coordinates at the reconstruction plane. $K(\xi)$ is a complex quantity that can be calculated on a number of planes at various distances $z_{r}$ from the point source (pinhole) in order to recover the threedimensional information of the sample, from a single twodimensional DIHM hologram. This operation is entirely done numerically and a full implementation would be equivalent to any attempt of non-approximated computation of the diffraction integral, namely extremely time consuming. To reduce the computation time of Eq. (1) without compromising the very demanding requirements of DIHM, Kreuzer [3] patented a procedure that cast this expression in a scalable convolution. The core of the patented procedure is a coordinate transformation that removes the non-linearity presented in the exponential term of Eq. (1); this transformation implies performing an interpolation over the contrast inline hologram, which leads to an intensity distribution on an equally spaced grid. The manipulation of both contrast hologram and Eq. (1) cast the latter in a new expression that can be computed via the use of fast Fourier transforms FFTs. Further details of the described procedure can be read in [3]; here for the purpose of this letter only the discrete version of the Kirchoff-Helmholtz transform is brought, which reads as:

$$
\begin{aligned}
& K\left(s \Delta \xi_{x}, t \Delta \xi_{y}\right)=\Delta x^{\prime} \Delta y^{\prime} \sum_{m=-M / 2}^{(M / 2)-1} \sum_{n=N / 2}^{(N / 2)-1} \tilde{I}^{\prime}\left(m \Delta x^{\prime}, n \Delta y^{\prime}\right) \\
& \times \exp \left[i k\left(m s \Delta x^{\prime} \Delta \xi_{x}+n t \Delta y^{\prime} \Delta \xi_{y}\right) / L\right]
\end{aligned}
$$

where $\tilde{I}^{\prime}\left(m \Delta x^{\prime}, n \Delta y^{\prime}\right)$ corresponds to the interpolated contrast hologram on $M \times N$ pixels, [3]. Equation (2) can be cast in a FFT if the sizes of pixels at the contrast hologram and the reconstruction planes are related through $\Delta \xi_{x}=\lambda L / M \Delta x^{\prime}$ and $\Delta \xi_{y}=\lambda L / N \Delta y^{\prime}$. This condition, which limits the performance of DIHM because it constrains the possible numerical apertures of the system, can be removed by introducing the substitution $\quad 2 m s=m^{2}+s^{2}-(m-s)^{2} \quad$ and $2 n t=n^{2}+t^{2}-(n-t)^{2}[3,9]$. For conciseness and without a lack of generality, in the equations to follow only one dimension would be considered, on the account that its extension to two dimensions can be straightforwardly done by the reader. Under this consideration, the above substitution transforms Eq. (2) in:

$$
\begin{aligned}
& K\left(s \Delta \xi_{x}\right)=\Delta x^{\prime} \exp \left[i k\left(s^{2} \Delta x^{\prime} \Delta \xi_{x}\right) / 2 L\right] \\
& \times \sum_{m=-M / 2}^{M / 2-1} \tilde{I}^{\prime}\left(m \Delta x^{\prime}\right) \exp \left[i k\left(m^{2} \Delta x^{\prime} \Delta \xi_{x}\right) / 2 L\right] . \\
& \times \exp \left[-i k\left((s-m)^{2} \Delta x^{\prime} \Delta \xi_{x}\right) / 2 L\right]
\end{aligned}
$$

This equation can be understood as a discrete convolution of the functions:

$$
f_{1}=\tilde{I}^{\prime}\left(m \Delta x^{\prime}\right) \exp \left[i k\left(m^{2} \Delta x^{\prime} \Delta \xi_{x}\right) / 2 L\right]
$$

and

$$
f_{2}=\exp \left[-i k\left(m^{2} \Delta x^{\prime} \Delta \xi_{x}\right) / 2 L\right]
$$

The reconstructed hologram can be therefore computed by the convolution theorem [3], which can be implemented via FFTs:

$$
\begin{aligned}
K\left(s \Delta \xi_{x}\right) & =\Delta x^{\prime} \exp \left[i k\left(s^{2} \Delta x^{\prime} \Delta \xi_{x}\right) / 2 L\right] \\
& \times F F T^{-1}\left\{F F T\left\{f_{1}\right\} \times F F T\left\{f_{2}\right\}\right\}
\end{aligned}
$$

Equation (6) allows for: i) reconstructing DIHM holograms with no restriction on the pixel sizes at the hologram and reconstruction plane and ii) accelerating its computation because it is FFT based and fully parallelizable.

The simplest approach to parallel computing can be the use of the power provided by the multi-core hardware of the GPUs in the CUDA ${ }^{\circledR}$ programming architecture [10]. CUDA $^{\circledR} \mathrm{C}$ is a programming language that allows for using the power of this architecture by means of defining $\mathrm{C}$ functions called kernels. The latter are concurrently executed by the multiple CUDA threads as defined by the programmer. Parallel computing is possible because each of the stream cores that are allocated inside the multiprocessors takes care of each thread. In a regular GPU, of the order of 8 multiprocessors, with 8 stream cores each, computing up to 64 threads at once is possible.

Additionally to the improvement provided by the use of the GPUs, an extra acceleration can be achieved on the computation of the two-dimensional version of equation (6); symmetry can be accounted in the calculation of the phase factor $\exp \left[i k\left(s^{2} \Delta x^{\prime} \Delta \xi_{x}+t^{2} \Delta y^{\prime} \Delta \xi_{y}\right) / 2 L\right]$ and the functions $f_{1}$ and $f_{2}$. These three terms depend on integers indices that take values from $-M / 2$ to $(M / 2)-1$ and from $-N / 2$ to $(N / 2)-1$, respectively. As for all the terms, the indices are square, and it suffices to compute these terms only for a quarter of the whole matrix over 
which the hologram is defined; the remaining three quarters can be computed via parallelized lookup tables.

For testing the performance of the accelerated reconstruction algorithm, there has been recorded and reconstructed a hologram from a monolayer of polystyrene beads with $1.10 \mu \mathrm{m}$ diameter each; the hologram was recorded on a CMOS camera $(2048 \times 2048$ pixels and $12.3 \times 12.3 \mathrm{~mm}^{2}$ ) at a distance $L=16.5 \mathrm{~mm}$ from the point source; the sample was placed at a distance of $80 \mu \mathrm{m}$ from the source. The recorded hologram has been pixel wise subtracted from the intensity with no sample present. This subtraction, called contrast hologram, does not carry information about the zeroth order of diffraction and gets rid of all the imperfection of the illumination.

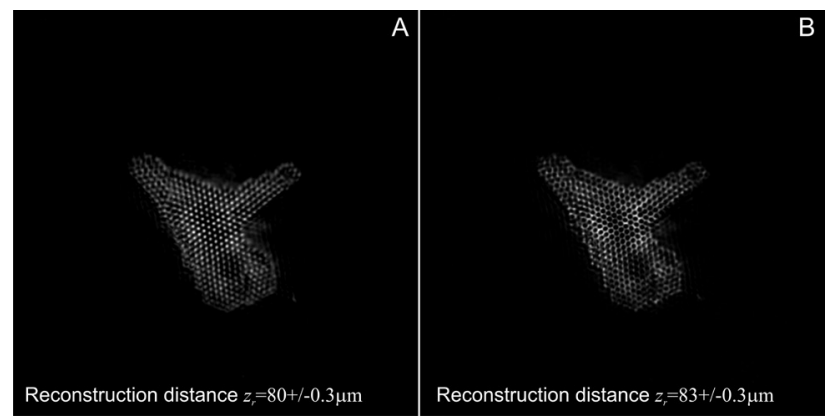

Fig. 1. Reconstruction of a DIHM hologram of a monolayer of polystyrene beads with $1.10 \mu \mathrm{m}$ diameter. Panel A shows the reconstruction at the object plane and panel $\mathrm{B}$ presents the reconstructed image half Talbot distance apart from panel A.

Panel A of Fig. 1, shows a reconstructed cluster with a perfect hexagonal packing of the spheres with some blurring and expansion of the lattice at the edges. The great quality of the reconstructions provided by the accelerated DIHM reconstruction algorithm, allows for studying characteristics of objects at the micrometre range, and for visualising in real space the valuable parameters of objects, as for this case, the hexagonal packing of the self-organised spheres. Further details on the reconstruction process can be read elsewhere [1-3,11].

Because DIHM has the capability of recording threedimensional information, it is possible to reconstruct images that show the self-imaging effect as the reconstruction distance is changed [11]; panel $\mathrm{B}$ shows a reversed contrast image reconstructed from the same hologram as panel $\mathrm{A}$; the distance between the reconstructed images of Fig. 1 is $3.0 \pm 0.3 \mu \mathrm{m}$. This value equals half of the Talbot distance [12]; for the used wavelength of $405 \mathrm{~nm}$ it leads to a pitch of $1.10 \pm 0.08 \mu \mathrm{m}$ for the periodic structure. This value is in great agreement with the diameter of the spheres reported by the manufacturer of $1.100 \pm 0.008 \mu \mathrm{m}$, so that the periodic structures are indeed closed-packed hexagons and the reconstruction algorithm can recover such valuable information. The results in Fig. 1 show that the acceleration of the reconstruction algorithm does not affect its performance, even in the case of very demanding DIHM holograms obtaining micrometre resolution with an important reduction of the reconstruction time, as shown below.

The reconstruction algorithm has been implemented in a personal computer powered with an Intel ${ }^{\circledR}$ Core $^{\mathrm{TM}} 2$ Quad running at $2.33 \mathrm{GHz}$ and $4 \mathrm{~GB}$ of RAM memory. The computer hosts a Geforce 9800GT graphics card with 112 stream cores, $512 \mathrm{MB}$ of local memory and running at $600 \mathrm{MHz}$; Table 1 summarises the results obtained as holograms of different sizes are reconstructed for accelerated and non accelerated algorithms.

Table 1. Number of reconstructed holograms in fps for different sizes of the input hologram for accelerated and non-accelerated algorithms.

\begin{tabular}{|c|c|c|}
\hline $\begin{array}{c}\text { Hologram size } \\
\text { (pixels) }\end{array}$ & $\begin{array}{c}\text { Number of } \\
\text { reconstructed } \\
\text { holograms (fps). } \\
\text { Accelerated method }\end{array}$ & $\begin{array}{c}\text { Number of } \\
\text { reconstructed } \\
\text { holograms (fps). Non } \\
\text { accelerated method }\end{array}$ \\
\hline $512 \times 512$ & 83 & 13 \\
\hline $1024 \times 1024$ & 32 & 3 \\
\hline $2048 \times 2048$ & 10 & 1 \\
\hline
\end{tabular}

Table 1 shows that the speed of the reconstruction algorithm has been raised up to 10 times for $2048 \times 2048$ holograms as compared with the previously implemented software [13]. This fact, added to the proved performance of the algorithm, is very promising for obtaining DIHM in real time, enabling its application in many fields of research and technology, as in-situ biology.

J. Garcia-Sucerquia acknowledges the helpful support of Prof. Hans Jurgen Kreuzer from Dalhousie University, during the time of the research done in the fascinating world of his invention, DIHM.

\section{References}

[1] J. Garcia-Sucerquia, W. Xu, S.K. Jericho, P. Klages, M.H. Jericho, H.J. Kreuzer, Appl. Opt. Lett. 45, 836 (2006)

[2] W. Xu, M.H. Jericho, H.J. Kreuzer, I.A. Meinertzhagen, Opt. Lett. 28, 164 (2003).

[3] H.J. Kreuzer, Holographic Microscope and Method of Hologram Reconstruction (US Patent 6.411.406 B1 2002).

[4] E.B. Ford, New Astro. 14(4), 406 (2009).

[5] T. Shimobaba, Y. Sato, J. Miura, M. Takenouchi, T. Ito, Opt. Exp. 16, 11776 (2008).

[6] U. Schnars, W. Juptner, Meas. Sci. Technol. 13 R85, 9 (2002).

[7] M. Sypek, C. Prokopowicz, M. Gorecki, Opt. Eng. 42, 3158 (2003).

[8] H.J. Kreuzer, K. Nakamura, A. Wierzbicki, H.W. Fink, H. Schmid, Ultramicroscopy 45, 381 (1992).

[9] L. Bluestein, IEEE. T on Audio and Electroacoustics. 18, 451 (1970).

[10] 2006-2010 NVIDIA Corporation, CUDA Zone (2010), http://developer.download.nvidia.com/compute/cuda/3_1/toolkit/docs/ NVIDIA CUDA C ProgrammingGuide 3.1.pdf

[11] J. Garcia-Sucerquia, D. C. Alvarez-Palacio, H.J. Kreuzer, Appl. Opt. 47, 4723 (2008).

[12] K. Patorski, Progr. Opt., E. Wolf, ed, 27, 3 (1989).

[13] H.J. Kreuzer, P. Klages, A software package for the reconstruction of digital in-line and other holograms (Helix Science Applications, Halifax, N.S., Canada 2006) 